\title{
Research of Private Cloud Resource Allocation with Multi-type User Based on Online Mechanism Design
}

\author{
Xiaolong $\mathrm{Ma}^{1,2}$, Lanjuan $\mathrm{Liu}^{1}$ \\ ${ }^{1)}$ School of Information Management and Engineering, Shanghai University of Finance and Economics, Shang hai, China \\ (xiaolongma@ @utc.zj.cn) \\ ${ }^{2)}$ Huzhou Teachers College, Huzhou City, Zhejiang Province, China
}

\begin{abstract}
The development of cloud computing prompts a wide interest in market-based resource allocation mechanism by which a cloud provider aims at efficiently allocating cloud resources among potential users. A problem that at present the private cloud resource allocation within the enterprise exists is how to allocate resources more efficiently to maximize the user value for cloud users (coming on-the-fly) in the dynamic environment. Online mechanisms extend the methods of mechanism design to dynamic environments with multiple agents and private information. This paper first constructs an online private cloud auction framew ork where users with multi-type task could come and leave on the fly. Secondly, for different types of users, this paper designs a bidding language and puts forward a online mechanism which guarantees participating users bid their true type and theoretical analysis the dominant strategy incentive compatible property of the mechanism.
\end{abstract}

Keywords - Online Mechanism Design, Private Cloud, Resource Allocation

$$
\begin{aligned}
& \text { 基于在线机制设计的多类型私有云资源分配研究 } \\
& \text { 马小龙 }{ }^{1,2} \text { 刘兰娟 }{ }^{1} \\
& \text { 1) 上海财经大学信息管理与工程学院, 上海, 中国 } \\
& \text { 2) 湖州师范学院商学院, 浙江, 中国 }
\end{aligned}
$$

摘 要 云计算的发展促进了基于市场机制的资源分配机制的广泛研究, 通过机制设计可以使云服务商更有效率的在其潜在的云 用户中有效地分配资源。目前企业内部的私有云资源分配存在的一个问题是对于处在随时来也可以随时走 (coming on-the-fly) 的动态 环境中的云用户, 如何更有效率的分配资源, 使用户的价值最大化。在线机制设计理论扩展了传统的机制设计方法, 更有效的适用于 具有多个代理人和私有信息的动态环境。本文首先构建了一个在线私有云拍卖框架, 支持能够随时来也可以随时走的不同用户的工作 任务要求。其次提出了一个能保证参与用户能够报真实类型的在线机制, 理论分析证明了该机制的占优策略激励兼容性质 (DSIC)。

关键词 在线机制设计, 私有云, 资源分配

\section{1. 概述}

根据云资源对用户的不同开放程度，云计算资源大致 可分为私有云、公有云。私有云只对某一组织内的用户开 放, 而公有云则对所有用户开放, 并且两类云的目标也不 完全一样。私有云更倾向于为组织内用户带来更多的价值, 而公有云则更倾向于为自身带来更大的效益[1]。对于私有 云的构建者, 如何使所有用户的价值最大化, 即社会福利 最大化是其追求的目标。云计算是一种新兴商业计算模型, 商业化的特性使其不断关注用户日益多样化的需求, 目标
旨在按需灵活合理分配资源, 提升数据中心资源利用率[2] [3]。利用经济学中市场机制具有调动个体积极性，激励资 源共享, 实现合理资源配置的特点, 将机制设计应用于商 业化特性的云计算环境中, 是一种非常重要的方法[4]。

在线机制设计扩展了传统机制设计方法从而适应具有 多代理和私有信息更动态的环境。在线机制设计的特点是 决策所依赖的信息类型是在线显示, 而且决策时没有掌握 用户未来的信息[5] [6]。

现有的研究主要集中在使用传统机设计机制。这种机 
制的优点是用户容易理解和使用简单。但是目前的问题是: (1) 私有云云用户的应用类型不同。例如有批量工作型任务 用户，有工作时间截止型任务用户以及资源侵略型任务用 户。(2) 用户可能虚报自己私有信息来提高自己的效用。(3) 私有云用户是随时来, 也可以随时走, 处于动态的环境。

针对这些问题，本文首先设计了一个多类型用户在线 私有云拍卖框架, 这个框架可以支持随时来, 也可以随时 走的不同类型的用户，如表一所示[7]。这个框架的特点是：

(1) 私有云在线拍卖可以在线进行。用户随时按照需要提 出自己的任务要求, 云服务商马上可以处理用户的要求。

(2) 每个用户的要求被提交到集中控制中心, 从而可以通 过本文设计的在线机制来保证每个用户报真实类型。

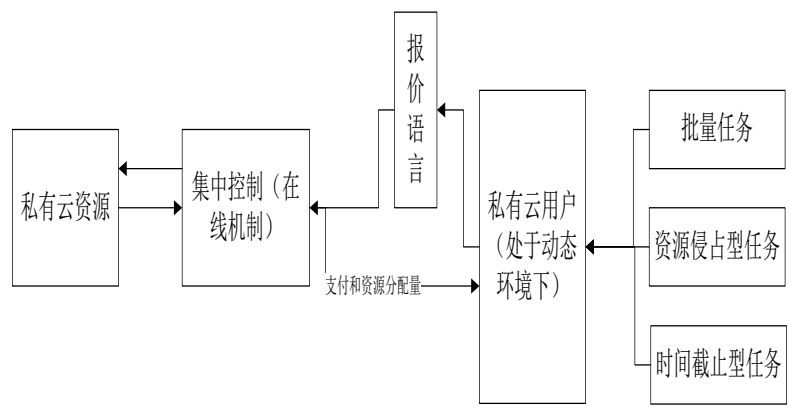

表一：私有云在线拍卖框架

\section{2. 模型}

（1）私有云资源提供商 (Supply): 考虑一个模型是 离散的、而且是有限时间步步骤 (比如按照单位时间划分) $t \in T$ 。提供商在无限的时间内, 提供大量的同种类型的计 算资源, 供给函数为 $S: T \rightarrow N_{0}^{+}, S(t)$ 为 $\mathrm{t}$ 时刻单位资 源的数量 (比如 CPU 处理时间), 每个时间步骤一个用户 可以至多分一个单位资源, 云计算资源属于易消失的资源, 意味着如果在某个时间段没有被分配该资源就会被浪费。

(2) 用户和用户的任务类型: 令 $I=\{1, \ldots, n\}$ 为所有 私有云用户的集合。一个用户在 $a_{i} \in T$ 时间到达, $d_{i} \in T$ 时间离开, 而且必须满足 $d_{i} \geq a_{i}$, 这个时间段内, 可以认 为用户是激活的, 能接受私有云服务。用户的任务可以分 为三种类型：批量工作类型 (batch)、资源侵占型 (Resource-aggressive) 和时间截止型（deadline）类型。

批量工作类型任务: 该类任务的特点是用户得到的价 值与分配到资源量成正比。

资源侵占型工作：这类工作任务的的特点是在具体的 时间段需要更多的资源, 用户可以获得更多的价值。

截止日期型工作 (Deadline): 该类工作的特点是用
户对资源的要求有时间限制, 截止时间到, 资源不满足 分配价值为 0 。

这三种价值函数都满足边际价值不增的性质, 也就是 给定价值向量 $v_{i}=<v_{i, 1}, v_{i, 2}, \ldots, v_{i, m_{i}}>$, 其中 $v_{i, k}$ 代表 $\mathrm{i}$ 用 户对第 $\mathrm{k}$ 个单位资源的边际价值, 满足 $v_{i, k} \geq 0$ 和 $v_{i, k+1} \leq v_{i, k}$ 。

（3）报价和机制: 令 $\hat{\theta}_{i}=\left\{\hat{a}_{i}, \hat{d}, \hat{v}_{i}\right\}_{\text {表示用户报告的 }}$ 类型。机制采纳用户的私有报告类型, 基于这些报告类型 来决定资源的分配和用户的支付。本文机制设计的目标就 是激励用户真实报告自己的类型。分配政策 $\pi_{i}^{\langle t>}\left(\hat{\theta} ; k^{<t>}\right)$ 表示在 $t \in T$ 时刻为每个用户 $i \in I$ 的分配, 其中 $k^{<t>}=\left(K_{1}^{\star}>\ldots k_{n}^{k}\right.$ 表示在 $\mathrm{t}$ 时刻拍卖开始之前用户分配 的总的资源量, 对于每个用户 $\mathrm{i}$ 在 $\mathrm{t}$ 时刻分配的资源量 $k_{i}^{<t>}=\sum_{t^{\prime}=\hat{a}_{i}}^{t-1} \pi_{i}^{\left\langle t^{\prime}\right\rangle}\left(\hat{\theta}_{i}, \hat{\theta}_{-i} \mid k^{\left\langle t^{\prime}\right\rangle}\right)$ 。在用户 $\mathrm{i}$ 报告的 $<\hat{a}_{i}, \hat{d}_{i}>$ 的时间段内，分配的总资源量表示为:

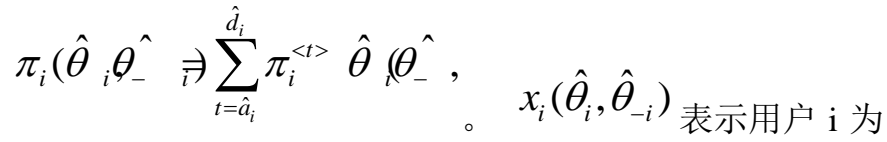
获得分配资源的支付。

（4）用户效用: 效用函数指的是用户从分配中获得的 价值减去支付给云服务商的费用, 计算公式为 $u_{i}\left(\hat{\theta} ; \theta_{i}\right) \sum_{k=1}^{\pi_{i}\left(\hat{\theta}_{i}, \hat{\theta} \theta_{i}\right)} v_{i, k}-x\left(\hat{\theta}, \hat{\theta} \mid \pi \hat{\theta}_{i} \hat{\theta}{ }_{y_{i}} \hat{\theta}\right.$ 。因为每个 用户都是自利的, 他们有可能为了最大化他们的效用, 可 能会虚报他们的个人私有类型。

（5）有限误报: 本文假设用户不能虚报早到的时间和 迟的离开时间, 即 $\hat{a}_{i} \geq a_{i}$ 和 $\hat{d}_{i} \leq d_{i}$, 本文认为用户报 $<\hat{a}_{i}, \hat{d}_{i}>_{\text {是合理的。 }}$

\section{3. 机制设计}

本文设计的机制如下:

(1) 分配政策:

第一步: 在每个 $t$ 时刻, 先使用贪心分配进行预分配。 第二步: 分别计算 $v_{i, k_{i}^{k t}+1}$ 和 $p_{-i, k_{i}^{t}+1}^{t}$, 如果满足公式

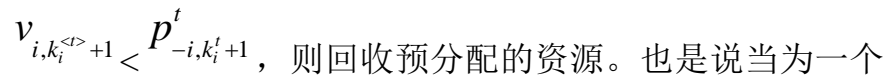
新增加的资源带来的边际价值小于为这个资源的支付, 那 
么分配这个资源不能增加价值, 所以就回收这个单位的云 资源。

（2）支付政策：当用户离开时，发生支付。假如在 $\mathrm{t}$ 时刻, 用户 $\mathrm{i}$ 被分配了 $\pi_{i}$ 个单位资源, 用户 $\mathrm{i}$ 需要的支付 是:

$$
x_{i}\left(\hat{\theta}_{i}, \hat{\theta}_{-i} \mid \pi_{i}\right)=\sum_{k=1}^{\pi_{i}} p_{-i, k}
$$

\section{4. 理论分析}

（1）定义 2:（阈值分配政策)。给定一个用户 $\mathrm{i}$,固 定 $<\hat{a}_{i}, \hat{d}_{i}>$ 和 $\hat{\theta}_{-i}$, 存在一个边际非递增阈值向量 $\mathrm{T}$, 这个向量 $\mathrm{T}$ 与用户报告的价值 $\hat{v}_{i}$ 相互独立, 对于 $\forall k$, $\hat{v}_{i}$, 当且仅当 $\hat{v}_{i, k} \geq \mathrm{T}_{k}, \pi_{i}\left(\hat{\theta}_{i}, \hat{\theta}_{-i}\right) \geq k$ 成立, 那么分配 政策 $\pi$ 就是阈值分配政策。

也可以说，一个阈值分配政策对于每个 $\mathrm{k}$ 有个间值 $\mathrm{T}_{k}$, 当且仅当用户 $\mathrm{i}$ 为第 $\mathrm{k}$ 个资源报告的价值至少为 $\mathrm{T}_{k}$ 时，用户 $\mathrm{i}$ 可以接受这 $\mathrm{k}$ 个资源。

引理 1: 对于给定可接受的 $<\hat{a}, \hat{d}>$ 和 $\hat{\theta}_{-i}$, 一个阈 值分配政策加上下面这个支付政策, $x_{i}\left(\hat{\theta}_{i}, \hat{\theta}_{-i}\right)=\sum_{k=1}^{\pi_{i}\left(\hat{\theta}_{i}, \hat{\theta}_{-i}\right)} \mathrm{T}_{k}$, 如果 $v_{i}$ 满足边际非递增, 那么 用户 $\mathrm{i}$ 报真正的价值 $v_{i}$ 是一个弱占优策略。

证明：用户 $\mathrm{i}$ 的效用可以定义以下： $u_{i}\left(\hat{\theta}_{i} ; \theta_{i}\right)=\sum_{k=1}^{\pi_{i}\left(\hat{\theta}_{i}, \hat{\theta}_{-i}\right)}\left(v_{i, k}-\mathrm{T}_{k}\right)$ 。因为 $\mathrm{T}$ 是独立于用户报 告的价值 $\hat{\mathrm{v}}_{i}$, 用户 $\mathrm{i}$ 仅仅通过改变分配政策 $\pi_{i}\left(\hat{\theta}_{i}, \hat{\theta}_{-i}\right)_{\text {来 }}$ 获利。因为 $\mathrm{T}_{k+1} \geq \mathrm{T}_{k}$ 和 $v_{i, k+1} \leq v_{i, k}$ 。通过定义 2 , 对于任 意的 $k \leq \pi_{i\left(\theta_{i}\right)}, \quad v_{i, k}-\mathrm{T}_{k} \geq 0$ 成立。假如用户 $\mathrm{i}$ 通过误报 得到了分配大于真实报的分配 $\pi_{i}\left(\hat{\theta}_{i}\right)>\pi_{i}\left(\theta_{i}\right)$, 因为 $\sum_{k=\pi_{i}\left(\hat{\theta}_{i}, \hat{\theta}_{i} * 1\right.}^{\pi_{i}\left(\hat{\theta}_{i}, \hat{\theta}_{i}\right)}\left(v_{i, k}-\mathrm{T}_{k}\right)<0$ ，所以有 $u_{i}\left(\hat{\theta}_{i} ; \theta_{i}\right)<u_{i}\left(\theta_{i}\right)$ 。同 理如果用户 $\mathrm{i}$ 通过误报得到了分配小于真实报的分配 $\pi_{i}\left(\hat{\theta}_{i}\right)<\pi_{i}\left(\theta_{i}\right)$, 因为 $\sum_{k=\pi_{i}}^{\pi_{i}\left(\hat{\theta}_{i}, \hat{\theta}_{i}, \hat{\theta}_{-i}\right)+1}\left(v_{i, k}-\mathrm{T}_{k}\right) \geq 0$, 所以有 $u_{i}\left(\hat{\theta}_{i} ; \theta_{i}\right)<u_{i}\left(\theta_{i}\right)$ 。用户如果误报对分配没有影响, 那么 效用也保持不变。通过以上证明, 对于用户 $\mathrm{i}$ 误报它的 价值是没有激励的。
引理 2: 给定非递增的边际价值, 本文设计的分配政 策是一个门槛政策, 在这里令 $\mathrm{T}_{k}=p_{-i, k}$ 。

证明:

情况一：令 $v_{i, k} \geq p_{-i, k}$ ，假如用户 $\mathrm{i}$ 对前 $\mathrm{k}$ 个单位的 资源有相同的边际价值, 比如: $v_{i, 1}=v_{i, 2}=\ldots=v_{i, k}$, 那 么在本机制的预分配阶段给定 $v_{i, k} \geq v_{-i, t}, t \in T_{i}$, 用户 $\mathrm{i}$ 都 会赢得拍卖。注意即使用户 $\mathrm{i}$ 赢得了这个拍卖, 因为拍卖 带来的价值小于或者等于 $v_{i, k}$, 用户 $\mathrm{i}$ 放弃了这次拍卖, 对 用户 $\mathrm{i}$ 来说这也不影响未来的拍卖, 因为在下个拍卖用户 $\mathrm{i}$ 报价依然高于其他用户。现在因为当 $j \leq k, p_{-i, j} \leq p_{-i, k}$, 在时间周期 $t \in T$ 内, 至少存在 $\mathrm{k}$ 次拍卖, 这些拍卖满足 $p_{-i, k} \geq v_{-i, t}$ ，因为 $v_{i, k} \geq p_{-i, k}$ ，用户 $\mathrm{i}$ 至少在机制的第一 阶段可以赢得 $\mathrm{k}$ 次拍卖。

进一步, 每次拍卖都赢了, 出清价格是 $p_{-i}^{t}$ 向量的前 $\mathrm{j}$ 个值之一, 在这里 $\mathrm{j}$ 是拍卖的次数。因为用户 $\mathrm{i}$ 满足 $v_{i, k} \geq v_{-i, t}$, 在第一阶段赢得了拍卖。无论何时在第一阶段 赢得拍卖, 就满足 $v_{i, k}=v_{i, j} \geq p_{-i, j}$, 因此在第二阶段就 不回收这个预分配的资源。实际上，价值满足非递增的 价值，对 $1 \leq j \leq k$ ，有 $v_{i, j} \geq v_{i, k}$ ，这样本机制的分配政 策将分配给用户至少 $\mathrm{k}$ 个单位的资源。

情 况二: 令 $v_{i, k}<p_{-i, k}$, 注意到 当 $\left(a_{i}+k-1\right) \leq t \leq d_{i}$ 时, 满足 $p_{-i, k} \leq p_{-i, k}^{<t>}$ 。也就是阈值 随着时间递减。这样对于任意 $\left(a_{i}+k-1\right) \leq t \leq d_{i}$, $v_{-i, k}<p_{-i, k}^{<t>}$ 成立。考虑一种情况, 在 $t_{k}$ 时刻, 第一阶段 分配了第 $\mathrm{k}$ 个单位资源。因为 $v_{-i, k}<p_{-i, k}^{<t>}$, 这个单位的 资源在第二阶段被回收了。因此最后的结果是分配了少 于 $\mathrm{k}$ 单位的资源。

引理 3: 给定有限误报, 和假设在给定的用户报告 的类型 $<\hat{a}_{i}, \hat{d}_{i}>$ 前提下, 报告真实价值 $\hat{v}_{i}=v_{i}$ 是一个占 优策略, 那么真实报告 $\hat{a}_{i}=a_{i}$ 和 $\hat{d}_{i}=d_{i}$ 是一个占优策 略。

证明: 给定用户报告的类型 $\hat{\theta}_{i}=<\hat{a}_{i}, \hat{d}_{i}, \hat{v}_{i}>$, 令 $p_{-i}^{<\hat{a}_{i}, \hat{d}_{i}>}$ 表示用户 $\mathrm{i}$ 不参与递增顺序的边际出清价格。通 
过报告类型 $\hat{\theta}_{i}$, 用户 $\mathrm{i}$ 被分配了 $\pi_{i}\left(\hat{\theta}_{i}\right)$ 个资源, 总的支 $\sum_{j=1}^{\pi_{i}\left(\hat{\theta}_{i}\right)} p_{-i, j}^{<\hat{a}_{i}, \hat{d}_{i}>}$

付是 $\sum_{j=1} p_{-i, j}$ 。对于每个用户 $\mathrm{i}$, 从真实类型 $\theta_{i}$ 到误报 类型 $\hat{\theta}_{i}$ 将造成以下两种情况之一:

(1) 误报的分配和真实的分配相等, 即 $\pi_{i}\left(\hat{\theta}_{i}\right)=\pi_{i}\left(\theta_{i}\right)$ 。用户误报对于边际出清价格 ${ }^{v_{-i, t}}$ 没有任 何影响, 但是仅仅增加了向量 $p_{-i}$ 的长度。特别是用户 $\mathrm{i}$ 有限误报 $\left(\hat{a}_{i} \geq a_{i}\right.$ 和 $\hat{d}_{i} \leq d_{i}$ ), 就有 $p_{-i}^{<\hat{a}_{i}, \hat{d}_{i}>}$ 包含了向量 $p_{-i}^{<a_{i}, d_{i}>}$ 元素的子集。因为通过定义可知这些向量是递增 的, 只要存在 $j \leq\left(\hat{d}_{i}-\hat{a}_{i}+1\right), p_{-i, j}^{<\hat{i}_{i}, \hat{d}_{i}>} \geq p_{-i, j}^{<a_{i}, d_{i}>}$ 满足。

(2) 如果误报的分配不等于真实的分配, 即 $\pi_{i}\left(\hat{\theta}_{i}\right) \neq \pi_{i}\left(\theta_{i}\right.$ 。首先 $\pi_{i}\left(\hat{\theta}_{i}\right)>\pi_{i}\left(\theta_{i}\right)$ 不可能发生。这是 因为边际出清价格一致, 但是用户参与的拍卖次数随着误 报减少, 本机制的第一阶段的分配可能变小。此外, 因为 $p_{-i, j}^{<\hat{a}_{i}, \hat{d}_{i}>} \geq p_{-i, j}^{<a_{i}, d_{i}>}$, 在第二阶段回收的可能性变小。这样 就可以保证 $\pi_{i}\left(\hat{\theta}_{i}\right)<\pi_{i}\left(\theta_{i}\right)$ 。

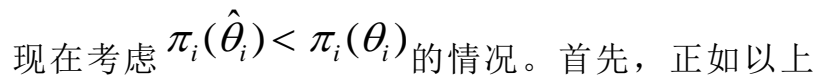
$\pi_{i}\left(\hat{\theta}_{i}\right)=\pi_{i}\left(\theta_{i}\right)$ 情 形, 可以知道 $\sum_{j=1}^{\pi_{i}\left(\hat{\theta}_{i}\right)} p_{-i, j}^{<a_{i}, d_{i}>} \leq \sum_{j=1}^{\pi_{i}\left(\hat{\theta}_{i}\right)} p_{-i, k}^{<\hat{a}_{i}, \hat{d}_{i}>}$

，这是因为用户误报到达和 离开时间赢得了更多的资源, 就要付出更多的费用。此外, 可以知道对于任意虚报分配 $\pi_{i}\left(\hat{\theta}_{i}\right)<\pi_{i}\left(\theta_{i}\right)$ 情形下, 真实 分配 $\pi_{i}\left(\theta_{i}\right)$ 是较好的选择, 否则报真实价值 $v_{i}$ 不再是占优 策略。因为误报导致了支付的费用更高一些, 用户就不能 从误报获得的额外分配获得收益。

定理 1: 给定非递增的边际价值和有限误报, 本文设 计的机制是占优策略激励兼容的。

证明: 本定理的证明可以从以上引理推导出来。根据 引理 1 和引理 2 , 对于任意对 $<\hat{a}_{i}, \hat{d}_{i}>$, 给定一个合适的 支付政策, 根据价值向量 $v_{i}$, 本机制的分配政策是说保证 报真实类型的策略。此外等式 (1) 的支付对应引理 2 的支 付, 因此, 可以保证用户说真话实现本机制。最后, 引理
3 通过展现对于真实的报价向量 $v_{i}$ ，用户不能通过误报到 达和离开时间来获益，从而完成了整个定理的证明。

\section{5. 结论}

本文首次尝试应用在线机制设计理论，解决用户随时 来也可以随时走的动态环境下企业私有云资源如何有效率 分配的问题。本文构建了一个在线私有云拍卖框架，针对 三种不同任务类型的用户，设计了一个能保证参与用户能 够报真实类型的在线机制, 理论分析证明了机制的 DSIC 性质。

\section{参考文献(References)}

[1] Jackie Fenn, Pete Basiliere, Kathy Harris, and Daryl C. Plummer. After the next big thing: The consequences of a cloud computing scenario. Technical report, Gartner Inc., May 2009.

[2] Kai Hwang, Geoffrey Fox, and Jack Dongarra. Distributed and Cloud Computing: From Parallel Processing to the Internet of Things. Morgan Kaufmann, 10/2011 2011.

[3] R. Buyya, C. S. Yeo, and S. Venugopal. Market-oriented cloud computing: Vision, hype, and reality for delivering it services as computing utilities. In Proceedings of 10th IEEE International Conference on High Performance Computing and Communications, 2008.

[4] Q. Zhang, E. Grses, R. Boutaba, and J. Xiao. Dynamic resource allocation for spot markets in clouds. In Proceedings of the 11th USENIX conference on Hot topics Hot-ICE, 2011.

[5] D.C. Parkes and S. Singh. An MDP-Based approach to Online Mechanism Design. In Proc. of NIPS'03, 2003.

[6] M. Armbrust, A. Fox, R. Griffith, A. D. Joseph, R. H. Katz, A. Konwinski, G. Lee, D. A. Patterson, A. Rabkin, I. Stoica, and M. Zaharia. Above the clouds: A berkeley view of cloud computing. Technical Report UCB/EECS-2009-28, Electrical Engineering and Computer Sciences, University of California at Berkeley, February 2009.

[7] M. Hajiaghayi, R. Kleinberg, M. Mahdian, and D.C. Parkes. Online auctions with re-usable goods. In Proc. of the 6th ACM Conf. onElectronic Commerce (EC'05), pages 165-174, 2005.

[8] D.C. Parkes. Online mechanisms. In N. Nisan, T. Roughgarden, E. Tardos, and V. Vazirani, editors, Algorithmic Game Theory, pages 411-439, 2007. 